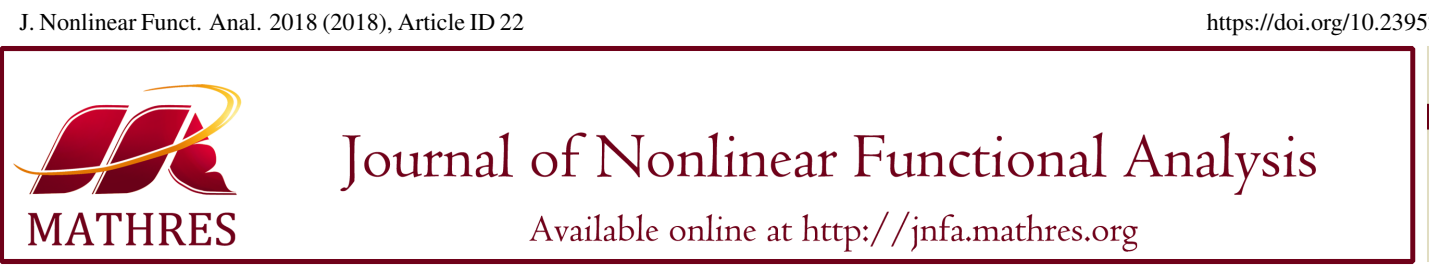

\title{
EXISTENCE OF POSITIVE SOLUTIONS FOR DIFFERENTIAL EQUATIONS INVOLVING RIEMANN-LIOUVILLE AND CAPUTO FRACTIONAL DERIVATIVES
}

\author{
YUNHONG LI*, YAN LI \\ School of Sciences, Hebei University of Science and Technology, \\ Shijiazhuang, 050018, Hebei, China
}

\begin{abstract}
In this paper, the existence of positive solutions is considered for differential equations involving the RiemannLiouville fractional derivative and the Caputo fractional derivative with $p$-Laplacian operators. Based on the monotone iterative technique, we obtain the existence of positive solutions for boundary value problems. Approximation of solutions is investigated. An example is also provided to illustrate the effectiveness of the main results.

Keywords. Positive solution; Riemann-Liouville fractional derivative; Caputo fractional derivative; $p$-Laplacian opeator; Monotone iterative technique.
\end{abstract}

2010 Mathematics Subject Classification. 34B15, 34B18.

\section{INTRODUCTION}

Fractional derivatives play an important role in the modeling of different physical and natural phenomena, such as, fluid mechanics, control systems and many other branches of engineering. Recently, there are many results on the Riemann-Liouville fractional differential equations with $p$-Laplacian (see [1]-[6]) and the Caputo fractional differential equations with $p$-Laplacian operators (see [7]-[16]).

In [1], Han, Lu and Zhang studied the existence of positive solutions for the following problem with the generalized $p$-Laplacian operator:

$$
\begin{array}{r}
D_{0^{+}}^{\beta}\left(\phi\left(D_{0^{+}}^{\alpha} u(t)\right)\right)=\lambda f(u(t)), 0<t<1, \\
u(0)=u^{\prime}(0)=u^{\prime}(1)=0, \phi\left(D_{0^{+}}^{\alpha} u(0)\right)=\left(\phi\left(D_{0^{+}}^{\alpha} u(1)\right)\right)^{\prime}=0,
\end{array}
$$

where $2<\alpha \leq 3,1<\beta \leq 2, D_{0^{+}}^{\alpha}$ and $D_{0^{+}}^{\beta}$ are the Riemann-Liouville fractional derivatives. Based on the properties of the Green's function and the Guo-Krasnosel'skii fixed point theorem on cones, some new existence results of at least one or two positive solutions are obtained for the boundary value problem.

\footnotetext{
${ }^{*}$ Corresponding author.
}

E-mail address: mathhong@126.com (Y. Li).

Received March 13, 2018; Accepted June 10, 2018. 
In [7], Chen, Liu and $\mathrm{Hu}$ investigated the existence of solutions by the coincidence degree for the Caputo fractional $p$-Laplacian equation

$$
\begin{array}{r}
D_{0^{+}}^{\beta} \phi_{p}\left(D_{0^{+}}^{\alpha} x(t)\right)=f\left(t, x(t), D_{0^{+}}^{\alpha} x(t)\right), 0<t<1, \\
D_{0^{+}}^{\alpha} x(0)=D_{0^{+}}^{\alpha} x(1)=0,
\end{array}
$$

where $D_{0^{+}}^{\beta}$ and $D_{0^{+}}^{\alpha}$ are the Caputo fractional derivatives, $0<\alpha, \beta \leq 1$ and $1<\alpha+\beta \leq 2, \phi_{p}(s)=$ $|s|^{p-2} s, p>1, f:[0,1] \times R^{2} \rightarrow R$ is continuous. They used $L u=D_{0^{+}}^{\beta} \phi_{p}\left(D_{0^{+}}^{\alpha} x(t)\right)$ with $D_{0^{+}}^{\alpha} x(0)=$ $D_{0^{+}}^{\alpha} x(1)=0$ and obtained $\operatorname{dim} \operatorname{Ker} L=1$.

Motivated by the above works, we investigate the existence of positive solutions for fractional differential equation:

$$
\left\{\begin{array}{l}
D_{0^{+}}^{\beta}\left[\phi_{p}\left({ }^{c} D_{0^{+}}^{\alpha} u(t)\right)\right]=f(t, u(t)), t \in(0,1), \\
{\left[\phi_{p}\left({ }^{c} D_{0^{+}}^{\alpha} u(0)\right)\right]^{(i)}=0, i=0,1, \cdots, m-1,} \\
\phi_{p}\left({ }^{c} D_{0^{+}}^{\alpha} u(1)\right)=0, \\
u^{(j)}(0)=0, j=2,3, \cdots, n, \\
u^{\prime}(1)=a \int_{0}^{1} h(t) u^{\prime}(t) \mathrm{d} t, u(0)=b \int_{0}^{1} h(t) u(t) \mathrm{d} t
\end{array}\right.
$$

where $1<n<\alpha \leq n+1,1<m<\beta \leq m+1$ and $m+n+1<\alpha+\beta \leq m+n+2, \phi_{p}(u)=|u|^{p-2} u, p>1$. $D_{0^{+}}^{\beta}$ is the Riemann-Liouville fractional derivative, ${ }^{c} D_{0^{+}}^{\alpha}$ is the Caputo fractional derivative. A function $u(t)$ is a positive solution of the boundary value problem (1.1) if and only if $u(t)$ satisfies the boundary value problem (1.1), and $u(t) \geq 0$ for $t \in[0,1]$. We use the monotone iterative technique to obtain the existence of positive solutions for boundary value problem (1.1). To the best of our knowledge, there are no results on the existence of positive solutions for mixed fractional $p$-Laplacian differential equations via the monotone iterative technique.

In this paper, we always suppose the following conditions are satisfied:

$\left(H_{1}\right) h(t):[0,1] \rightarrow[0, \infty)$ with $h \in C[0,1]$ and $f(t, u):[0,1] \times[0, \infty) \rightarrow[0, \infty)$ is continuous;

$\left(H_{2}\right) a>0, b>0,0<a \int_{0}^{1} h(t) \mathrm{d} t<1,0<b \int_{0}^{1} h(t) \mathrm{d} t<1$, and

$$
(\alpha-1) \int_{0}^{1} t h(t) \mathrm{d} t>\int_{0}^{1} h(t) \mathrm{d} t>0 .
$$

\section{PRELIMINARIES}

To show the main result of this paper, we give the following some basic definitions, which can be found in $[18,19]$.

Definition 2.1. The fractional integral of order $\alpha>0$ of a function $y:(0,+\infty) \rightarrow \mathbb{R}$ is given by

$$
I_{0+}^{\alpha} y(t)=\frac{1}{\Gamma(\alpha)} \int_{0}^{t}(t-s)^{\alpha-1} y(s) \mathrm{d} s,
$$

provided that the right side is pointwise defined on $(0,+\infty)$, where

$$
\Gamma(\alpha)=\int_{0}^{+\infty} e^{-x} x^{\alpha-1} \mathrm{~d} x
$$

Definition 2.2. For a continuous function $y:(0,+\infty) \rightarrow \mathbb{R}$, the Caputo derivative of fractional order $\alpha>0$ is defined as

$$
D_{0+}^{\alpha} y(t)=\frac{1}{\Gamma(n-\alpha)} \int_{0}^{t}(t-s)^{n-\alpha-1} y^{(n)}(s) \mathrm{d} s
$$


where $n=[\alpha]+1$, provided that the right side is pointwise defined on $(0,+\infty)$.

Definition 2.3. For a continuous function $y:(0,+\infty) \rightarrow \mathbb{R}$, the Riemann-Liouville derivative of fractional order $\alpha>0$ is defined as

$$
D_{0+}^{\alpha} y(t)=\frac{1}{\Gamma(n-\alpha)}\left(\frac{\mathrm{d}}{\mathrm{d} t}\right)^{n} \int_{0}^{t}(t-s)^{n-\alpha-1} y(s) \mathrm{d} s
$$

where $n=[\alpha]+1$, provided that the right side is pointwise defined on $(0,+\infty)$.

The following lemmas play an important role in this paper.

Lemma 2.4. The boundary value problem (1.1) is equivalent to the following equation:

$$
u(t)=d_{0}+d_{1} t-\frac{1}{\Gamma(\alpha)} \int_{0}^{t}(t-s)^{\alpha-1} v(s) \mathrm{d} s,
$$

where

$$
\begin{gathered}
d_{0}=d_{1} \frac{b \int_{0}^{1} t h(t) \mathrm{d} t}{1-b \int_{0}^{1} h(t) \mathrm{d} t}-\frac{b \int_{0}^{1} h(t) \int_{0}^{t}(t-s)^{\alpha-1} v(s) \mathrm{d} s \mathrm{~d} t}{\left[1-b \int_{0}^{1} h(t) \mathrm{d} t\right] \Gamma(\alpha)}, \\
d_{1}=\frac{\int_{0}^{1}(1-s)^{\alpha-2} v(s) \mathrm{d} s}{\left[1-a \int_{0}^{1} h(t) \mathrm{d} t\right] \Gamma(\alpha-1)}-\frac{a \int_{0}^{1} h(t) \int_{0}^{t}(t-s)^{\alpha-2} v(s) \mathrm{d} s \mathrm{~d} t}{\left[1-a \int_{0}^{1} h(t) \mathrm{d} t\right] \Gamma(\alpha-1)}, \\
v(s)=\phi_{q}\left(\int_{0}^{1} H(s, \tau) f(\tau, u(\tau)) \mathrm{d} \tau\right), \\
H(s, \tau)=\frac{1}{\Gamma(\beta)}\left\{\begin{array}{l}
(s-s \tau)^{\beta-1}-(s-\tau)^{\beta-1}, 0 \leq \tau \leq s \leq 1, \\
(s-s \tau)^{\beta-1}, 0 \leq s \leq \tau \leq 1 .
\end{array}\right.
\end{gathered}
$$

$\phi_{q}(s)$ is the inverse function of $\phi_{p}(s)$, a.e., $\phi_{q}(s)=|s|^{q-2} s, \frac{1}{p}+\frac{1}{q}=1, u(t) \in C[0,1]$.

Proof. From $D_{0^{+}}^{\beta}\left[\phi_{p}\left({ }^{c} D_{0^{+}}^{\alpha} u(t)\right)\right]=f(t, u(t))$, we get

$$
\phi_{p}\left({ }^{c} D_{0^{+}}^{\alpha} u(t)\right)=\frac{1}{\Gamma(\beta)} \int_{0}^{t}(t-\tau)^{\beta-1} f(\tau, u(\tau)) \mathrm{d} \tau+c_{1} t^{\beta-1}+c_{2} t^{\beta-2}+\cdots+c_{m+1} t^{\beta-m-1} .
$$

In view of $\left[\phi_{p}\left({ }^{c} D_{0^{+}}^{\alpha} u(0)\right)\right]^{(i)}=0, i=0,1, \cdots, m-1$, we get $c_{2}=\cdots=c_{m+1}=0$, i.e.,

$$
\phi_{p}\left({ }^{c} D_{0^{+}}^{\alpha} u(t)\right)=\frac{1}{\Gamma(\beta)} \int_{0}^{t}(t-\tau)^{\beta-1} f(\tau, u(\tau)) \mathrm{d} \tau+c_{1} t^{\beta-1} .
$$

Condition $\phi_{p}\left({ }^{c} D_{0^{+}}^{\alpha} u(1)\right)=0$ implies that

$$
c_{1}=-\frac{1}{\Gamma(\beta)} \int_{0}^{1}(1-\tau)^{\beta-1} f(\tau, u(\tau)) \mathrm{d} \tau
$$

It follows that

$$
\phi_{p}\left({ }^{c} D_{0^{+}}^{\alpha} u(t)\right)=-\int_{0}^{1} H(t, \tau) f(\tau, u(\tau)) \mathrm{d} \tau .
$$

Hence,

$$
{ }^{c} D_{0^{+}}^{\alpha} u(t)=-\phi_{q}\left(\int_{0}^{1} H(t, \tau) f(\tau, u(\tau)) \mathrm{d} \tau\right) .
$$

Letting

$$
v(t)=\phi_{q}\left(\int_{0}^{1} H(t, \tau) f(\tau, u(\tau)) \mathrm{d} \tau\right)
$$


we arrive at

$$
u(t)=-\frac{1}{\Gamma(\alpha)} \int_{0}^{t}(t-s)^{\alpha-1} v(s) \mathrm{d} s+d_{0}+d_{1} t+d_{2} t^{2}+\cdots+d_{n} t^{n}
$$

Conditions $u^{(j)}(0)=0, j=2, \cdots, n$, imply that $d_{2}=\cdots=d_{n}=0$, i.e.,

$$
u(t)=-\frac{1}{\Gamma(\alpha)} \int_{0}^{t}(t-s)^{\alpha-1} v(s) \mathrm{d} s+d_{0}+d_{1} t,
$$

which in turn yields that

$$
u^{\prime}(t)=-\frac{1}{\Gamma(\alpha-1)} \int_{0}^{t}(t-s)^{\alpha-2} v(s) \mathrm{d} s+d_{1}
$$

Condition $u^{\prime}(1)=a \int_{0}^{1} h(t) u^{\prime}(t) \mathrm{d} t$ implies that

$$
d_{1}=\frac{\int_{0}^{1}(1-s)^{\alpha-2} v(s) \mathrm{d} s}{\left[1-a \int_{0}^{1} h(t) \mathrm{d} t\right] \Gamma(\alpha-1)}-\frac{a \int_{0}^{1} h(t) \int_{0}^{t}(t-s)^{\alpha-2} v(s) \mathrm{d} s \mathrm{~d} t}{\left[1-a \int_{0}^{1} h(t) \mathrm{d} t\right] \Gamma(\alpha-1)} .
$$

From $u(0)=b \int_{0}^{1} h(t) u(t) \mathrm{d} t$, we get

$$
d_{0}=d_{1} \frac{b \int_{0}^{1} t h(t) \mathrm{d} t}{1-b \int_{0}^{1} h(t) \mathrm{d} t}-\frac{b \int_{0}^{1} h(t) \int_{0}^{t}(t-s)^{\alpha-1} v(s) \mathrm{d} s \mathrm{~d} t}{\left[1-b \int_{0}^{1} h(t) \mathrm{d} t\right] \Gamma(\alpha)} .
$$

The proof is complete.

Lemma 2.5. The function $u(t) \in C[0,1]$ defined by (2.1) is non-decreasing and non-negative.

Proof. Since

$$
u(t)=d_{0}+d_{1} t-\frac{1}{\Gamma(\alpha)} \int_{0}^{t}(t-s)^{\alpha-1} v(s) \mathrm{d} s,
$$

we find that

$$
\begin{aligned}
u^{\prime}(t) & =d_{1}-\frac{\int_{0}^{t}(t-s)^{\alpha-2} v(s) \mathrm{d} s}{\Gamma(\alpha-1)} \\
& =\frac{\int_{0}^{1}(1-s)^{\alpha-2} v(s) \mathrm{d} s}{\left[1-a \int_{0}^{1} h(t) \mathrm{d} t\right] \Gamma(\alpha-1)}-\frac{a \int_{0}^{1} h(t) \int_{0}^{t}(t-s)^{\alpha-2} v(s) \mathrm{d} s \mathrm{~d} t}{\left[1-a \int_{0}^{1} h(t) \mathrm{d} t\right] \Gamma(\alpha-1)}-\frac{\int_{0}^{t}(t-s)^{\alpha-2} v(s) \mathrm{d} s}{\Gamma(\alpha-1)} \\
& \geq \frac{\int_{0}^{1}(1-s)^{\alpha-2} v(s) \mathrm{d} s}{\Gamma(\alpha-1)}-\frac{\int_{0}^{t}(t-s)^{\alpha-2} v(s) \mathrm{d} s}{\Gamma(\alpha-1)} \geq 0 .
\end{aligned}
$$

Hence, we obtain that $u(t)$ is non-decreasing. It follows that $\min _{t \in[0,1]} u(t)=u(0)$. Since

$$
\begin{aligned}
d_{1} & =\frac{\int_{0}^{1}(1-s)^{\alpha-2} v(s) \mathrm{d} s}{\left[1-a \int_{0}^{1} h(t) \mathrm{d} t\right] \Gamma(\alpha-1)}-\frac{a \int_{0}^{1} h(t) \int_{0}^{t}(t-s)^{\alpha-2} v(s) \mathrm{d} s \mathrm{~d} t}{\left[1-a \int_{0}^{1} h(t) \mathrm{d} t\right] \Gamma(\alpha-1)} \\
& \geq \frac{\int_{0}^{1}(1-s)^{\alpha-2} v(s) \mathrm{d} s}{\Gamma(\alpha-1)}
\end{aligned}
$$


we find that

$$
\begin{aligned}
u(0) & =d_{0}=d_{1} \frac{b \int_{0}^{1} t h(t) \mathrm{d} t}{1-b \int_{0}^{1} h(t) \mathrm{d} t}-\frac{b \int_{0}^{1} h(t) \int_{0}^{t}(t-s)^{\alpha-1} v(s) \mathrm{d} s \mathrm{~d} t}{\left[1-b \int_{0}^{1} h(t) \mathrm{d} t\right] \Gamma(\alpha)} \\
& \geq \frac{b \int_{0}^{1} t h(t) \mathrm{d} t \int_{0}^{1}(1-s)^{\alpha-2} v(s) \mathrm{d} s}{\left[1-b \int_{0}^{1} h(t) \mathrm{d} t\right] \Gamma(\alpha-1)}-\frac{b \int_{0}^{1} h(t) \mathrm{d} t \int_{0}^{1}(1-s)^{\alpha-1} v(s) \mathrm{d} s}{\left[1-b \int_{0}^{1} h(t) \mathrm{d} t\right] \Gamma(\alpha)} \\
& \geq \frac{b\left[(\alpha-1) \int_{0}^{1} t h(t) \mathrm{d} t-\int_{0}^{1} h(t) \mathrm{d} t\right]}{\left[1-b \int_{0}^{1} h(t) \mathrm{d} t\right] \Gamma(\alpha)} \int_{0}^{1}(1-s)^{\alpha-1} v(s) \mathrm{d} s \geq 0 .
\end{aligned}
$$

The proof is complete.

Lemma 2.6. [17] The function $H(s, \tau)$ defined by $(2.5)$ is continuous on $[0,1] \times[0,1]$ and satisfies

$$
\frac{s^{\beta-1}(1-s) \tau(1-\tau)^{\beta-1}}{\Gamma(\beta)} \leq H(s, \tau) \leq \frac{\tau(1-\tau)^{\beta-1}}{\Gamma(\beta-1)} \text { for } s, \tau \in[0,1]
$$

\section{Main RESUlts}

Let the Banach space $E=C[0,1]$ be endowed with the norm $\|u\|=\max _{t \in[0,1]}|u(t)|$. Define the cone $P$ by $P=\{u \in E, u(t) \geq 0\}$. And define the operator $T: P \rightarrow E$,

$$
T u(t)=d_{0}+d_{1} t-\frac{1}{\Gamma(\alpha)} \int_{0}^{t}(t-s)^{\alpha-1} v(s) \mathrm{d} s .
$$

where $d_{0}, d_{1}, v(s)$ are defined by (2.2)-(2.4). Obviously, $u(t)$ is a solution of problem (1.1) if and only if $u$ is a fixed point of $T$.

Theorem 3.1. Assume that there exists $r>0$ such that

(C1) $f\left(t, u_{1}\right) \leq f\left(t, u_{2}\right), \forall 0 \leq t \leq 1,0 \leq u_{1} \leq u_{2} \leq r$;

(C2) $\max _{t \in[0,1]} f(t, r) \leq M$, where

$$
\begin{gathered}
M=(r L)^{p-1} \frac{\Gamma(\beta-1)}{B(2, \beta)}-1>0, \\
L=\left[1-a \int_{0}^{1} h(t) \mathrm{d} t\right]\left[1-b \int_{0}^{1} h(t) \mathrm{d} t\right] \Gamma(\alpha) .
\end{gathered}
$$

Then problem (1.1) has two positive solutions $w^{*}$ and $v^{*}$ such that

$$
\begin{gathered}
0 \leq w^{*}<r, 0 \leq v^{*}<r . \\
w^{*}=\lim _{k \rightarrow+\infty} w_{k}, v^{*}=\lim _{k \rightarrow+\infty} v_{k} . \\
w_{k}=T w_{k-1}, v_{k}=T v_{k-1}, k=1,2, \cdots . \\
w_{0}=\left(\frac{M}{M+1}\right)^{q-1} r, v_{0}=0 .
\end{gathered}
$$

Proof. The proof is split into three steps.

Step 1. We prove that $T: P \rightarrow P$ is completely continuous.

For any $u \in P$, we find from (2.6) that

$$
T u(t) \geq T u(0)=d_{0} \geq 0 .
$$


It follows that $T: P \rightarrow P$. Obviously, $T$ is continuous for the continuity of $f(t, u)$. Let $\Omega \subset P$ be bounded, that is, there exists a positive constant $\delta$, for any $u \in \Omega, u(t) \leq \delta$. For any $u \in \Omega$, we take $\lambda=\max _{0 \leq t \leq 1,0 \leq u \leq \delta} f(t, u)+1$. It follows that

$$
\begin{aligned}
v(s) & =\phi_{q}\left(\int_{0}^{1} H(s, \tau) f(\tau, u(\tau)) \mathrm{d} \tau\right) \\
& \leq \phi_{q}\left(\lambda \int_{0}^{1} \frac{\tau(1-\tau)^{\beta-1}}{\Gamma(\beta-1)} \mathrm{d} \tau\right)=\left(\frac{\lambda B(2, \beta)}{\Gamma(\beta-1)}\right)^{q-1} .
\end{aligned}
$$

Therefore, we get

$$
\begin{aligned}
T u(t)= & d_{0}+d_{1} t-\frac{\int_{0}^{t}(1-s)^{\alpha-1} v(s) \mathrm{d} s}{\Gamma(\alpha)} \\
= & \left(\frac{\int_{0}^{1}(1-s)^{\alpha-2} v(s) \mathrm{d} s}{\left[1-a \int_{0}^{1} h(t) \mathrm{d} t\right] \Gamma(\alpha-1)}-\frac{a \int_{0}^{1} h(t) \int_{0}^{t}(t-s)^{\alpha-2} v(s) \mathrm{d} s \mathrm{~d} t}{\left[1-a \int_{0}^{1} h(t) \mathrm{d} t\right] \Gamma(\alpha-1)}\right)\left\{\frac{b \int_{0}^{1} t h(t) \mathrm{d} t}{1-b \int_{0}^{1} h(t) \mathrm{d} t}+t\right\} \\
& -\frac{b \int_{0}^{1} h(t) \int_{0}^{t}(t-s)^{\alpha-1} v(s) \mathrm{d} s \mathrm{~d} t}{\left[1-b \int_{0}^{1} h(t) \mathrm{d} t\right] \Gamma(\alpha)}-\frac{\int_{0}^{t}(1-s)^{\alpha-1} v(s) \mathrm{d} s}{\Gamma(\alpha)} \\
\leq & \left(\frac{\int_{0}^{1}(1-s)^{\alpha-2} v(s) \mathrm{d} s}{\left[1-a \int_{0}^{1} h(t) \mathrm{d} t\right] \Gamma(\alpha-1)}\right)\left\{\frac{b \int_{0}^{1} t h(t) \mathrm{d} t}{1-b \int_{0}^{1} h(t) \mathrm{d} t}+1\right\} \\
\leq & \left(\frac{\lambda B(2, \beta)}{\Gamma(\beta-1)}\right)^{q-1}\left(\frac{1}{\left[1-a \int_{0}^{1} h(t) \mathrm{d} t\right]\left[1-b \int_{0}^{1} h(t) \mathrm{d} t\right] \Gamma(\alpha)}\right) .
\end{aligned}
$$

Hence, $T(\Omega)$ is uniformly bounded.

Next, we prove that $T(\Omega)$ is equicontinuous. For each $u \in \Omega$, if $0 \leq t_{1}<t_{2} \leq 1$, then

$$
\begin{aligned}
& \left|(T u)\left(t_{2}\right)-(T u)\left(t_{1}\right)\right| \\
= & \left|d_{1} t_{1}-\frac{\int_{0}^{t_{1}}(1-s)^{\alpha-1} v(s) \mathrm{d} s}{\Gamma(\alpha)}-d_{1} t_{2}+\frac{\int_{0}^{t_{2}}(1-s)^{\alpha-1} v(s) \mathrm{d} s}{\Gamma(\alpha)}\right| \\
\leq & d_{1}\left|t_{1}-t_{2}\right|+\left|\frac{\int_{0}^{t_{1}}(1-s)^{\alpha-1} v(s) \mathrm{d} s}{\Gamma(\alpha)}-\frac{\int_{0}^{t_{2}}(1-s)^{\alpha-1} v(s) \mathrm{d} s}{\Gamma(\alpha)}\right| \\
\leq & d_{1}\left|t_{1}-t_{2}\right|+\frac{\int_{t_{1}}^{t_{2}}(1-s)^{\alpha-1} v(s) \mathrm{d} s}{\Gamma(\alpha)} \\
\leq & d_{1}\left|t_{1}-t_{2}\right|+\left(\frac{\lambda B(2, \beta)}{\Gamma(\beta-1)}\right)^{q-1} \frac{\left|t_{1}-t_{2}\right|}{\Gamma(\alpha)},
\end{aligned}
$$

where

$$
\begin{aligned}
d_{1} & =\frac{\int_{0}^{1}(1-s)^{\alpha-2} v(s) \mathrm{d} s}{\left[1-a \int_{0}^{1} h(t) \mathrm{d} t\right] \Gamma(\alpha-1)}-\frac{a \int_{0}^{1} h(t) \int_{0}^{t}(t-s)^{\alpha-2} v(s) \mathrm{d} s \mathrm{~d} t}{\left[1-a \int_{0}^{1} h(t) \mathrm{d} t\right] \Gamma(\alpha-1)} \\
& \leq \frac{\int_{0}^{1}(1-s)^{\alpha-2} v(s) \mathrm{d} s}{\left[1-a \int_{0}^{1} h(t) \mathrm{d} t\right] \Gamma(\alpha-1)} \\
& \leq\left(\frac{\lambda B(2, \beta)}{\Gamma(\beta-1)}\right)^{q-1} \frac{1}{\left[1-a \int_{0}^{1} h(t) \mathrm{d} t\right] \Gamma(\alpha)} .
\end{aligned}
$$


Therefore, $T(\Omega)$ is equicontinuous. Applying the Arzelá-Ascoli theorem, we conclude that $T$ is a completely continuous operator.

Step 2. Let $\bar{P}_{r}=\{u \in P: 0 \leq\|u\| \leq r\}$, then we prove $T: \bar{P}_{r} \rightarrow \bar{P}_{r}$.

For any $u \in \bar{P}_{r}$, one has

$$
\begin{aligned}
T u(t) \leq & T u(1)=d_{0}+d_{1}-\frac{\int_{0}^{1}(1-s)^{\alpha-1} v(s) \mathrm{d} s}{\Gamma(\alpha)} \\
= & \left(\frac{\int_{0}^{1}(1-s)^{\alpha-2} v(s) \mathrm{d} s}{\left[1-a \int_{0}^{1} h(t) \mathrm{d} t\right] \Gamma(\alpha-1)}-\frac{a \int_{0}^{1} h(t) \int_{0}^{t}(t-s)^{\alpha-2} v(s) \mathrm{d} s \mathrm{~d} t}{\left[1-a \int_{0}^{1} h(t) \mathrm{d} t\right] \Gamma(\alpha-1)}\right)\left\{\frac{b \int_{0}^{1} t h(t) \mathrm{d} t}{1-b \int_{0}^{1} h(t) \mathrm{d} t}+1\right\} \\
& -\frac{b \int_{0}^{1} h(t) \int_{0}^{t}(t-s)^{\alpha-1} v(s) \mathrm{d} s \mathrm{~d} t}{\left[1-b \int_{0}^{1} h(t) \mathrm{d} t\right] \Gamma(\alpha)}-\frac{\int_{0}^{1}(1-s)^{\alpha-1} v(s) \mathrm{d} s}{\Gamma(\alpha)} \\
\leq & \left(\frac{\int_{0}^{1}(1-s)^{\alpha-2} v(s) \mathrm{d} s}{\left[1-a \int_{0}^{1} h(t) \mathrm{d} t\right] \Gamma(\alpha-1)}\right)\left\{\frac{b \int_{0}^{1} t h(t) \mathrm{d} t}{1-b \int_{0}^{1} h(t) \mathrm{d} t}+1\right\} \\
\leq & \left(\frac{M B(2, \beta)}{\Gamma(\beta-1)}\right)^{q-1}\left(\frac{\int_{0}^{1}(1-s)^{\alpha-2} \mathrm{~d} s}{\left[1-a \int_{0}^{1} h(t) \mathrm{d} t\right] \Gamma(\alpha-1)}\right)\left\{\frac{b \int_{0}^{1} t h(t) \mathrm{d} t}{1-b \int_{0}^{1} h(t) \mathrm{d} t}+1\right\} \\
\leq & \left(\frac{M B(2, \beta)}{\Gamma(\beta-1)}\right)^{q-1} \frac{1}{\left[1-a \int_{0}^{1} h(t) \mathrm{d} t\right] \Gamma(\alpha)} \frac{1-b \int_{0}^{1} h(t) \mathrm{d} t}{1} \\
= & \left(\frac{B(2, \beta)}{\Gamma(\beta-1)}\right)^{q-1} \frac{M^{q-1}}{L}=\left(\frac{M}{M+1}\right)^{q-1} r<r .
\end{aligned}
$$

Consequently, we get that $T: \bar{P}_{r} \rightarrow \bar{P}_{r}$.

Step 3. We prove that $w^{*}$ and $v^{*}$ are two positive solutions of problem (1.1).

Note that $w_{0}(t)=\left(\frac{M}{M+1}\right)^{q-1} r$. It is obvious, $w_{0}(t) \in \bar{P}_{r}$. Let $w_{k+1}(t)=T w_{k}(t), k=0,1,2, \cdots$. Since $T: \bar{P}_{r} \rightarrow \bar{P}_{r}$, we get $w_{k}(t) \in \bar{P}_{r}$. In view of (3.3), we get $T w_{0}(t) \leq\left(\frac{M}{M+1}\right)^{q-1} r=w_{0}(t)$, that is, $w_{1}(t) \leq w_{0}(t)$, Using the definition of $T$, we obtain

$$
w_{2}(t)=T w_{1}(t) \leq T w_{0}(t)=w_{1}(t) .
$$

By induction, we obtain

$$
w_{k+1}(t) \leq w_{k}(t), k=0,1,2, \cdots .
$$

Thus, we get $w^{*} \in \bar{P}_{r}$ such that $w_{k} \rightarrow w^{*}$. Applying the continuity of $T$ and $w_{k+1}(t)=T w_{k}(t)$, we get $w^{*}(t)=T w^{*}(t)$. Hence, $w^{*}(t)$ is a positive solution of problem (1.1). Note that $v_{0}(t)=0$. It is obvious, $v_{0}(t) \in \bar{P}_{r}$. Let $v_{k+1}(t)=T v_{k}(t), k=0,1,2, \cdots$. Since $T: \bar{P}_{r} \rightarrow \bar{P}_{r}$, we get that $v_{k}(t) \in \bar{P}_{r}$. In view of Lemma 2.2, we get $T v_{0}(t) \geq 0=v_{0}(t)$, that is, $v_{1}(t) \geq v_{0}(t)$. From the definition of $T$, we obtain

$$
v_{2}(t)=T v_{1}(t) \geq T v_{0}(t)=v_{1}(t) .
$$

By induction, we obtain

$$
v_{k+1}(t) \geq v_{k}(t), k=0,1,2, \cdots .
$$

Thus, we get $v^{*} \in \bar{P}_{r}$ such that $v_{k} \rightarrow v^{*}$. Applying the continuity of $T$ and $v_{k+1}(t)=T v_{k}(t)$, we get $v^{*}(t)=T v^{*}(t)$. Hence $v^{*}(t)$ is a positive solution of problem (1.1). 
Remark 3.2. The positive solutions $w^{*}(t)$ and $v^{*}(t)$ of problem (1.1) in Theorem 3.1 may coincide, and in this case, problem (1.1) has a positive solution in $\bar{P}_{r}$.

Finally, we give a simple example to explain the main theorem.

Example 3.3. For problem (1.1), let $\alpha=2.6, \beta=2.9, a=10, b=9, p=1.5, r=10, h(t)=$ $0.1 t, f(t, u)=5 \cos t+\sin t+t u$. From a direct calculation, we get

$$
\begin{gathered}
q=3, \int_{0}^{1} h(t) \mathrm{d} s=0.05, L=0.393147, M=20.568088 . \\
\max _{t \in[0,1]} f(t, r)<5+1+10=16<M .
\end{gathered}
$$

Then all the conditions of Theorem 3.1 are satisfied. Hence, we get that the problem has two positive solutions $w^{*}$ and $v^{*}$.

\section{Acknowledgements}

This work was supported by the Foundation of Hebei Education Department (QN2018104) and Hebei Province Natural Science Fund (A2018208171).

\section{REFERENCES}

[1] Z. Han, H. Lu, C. Zhang, Positive solutions for eigenvalue problems of fractional differential equation with generalized p-Laplacian, Appl. Math. Comput. 257 (2015), 526-536.

[2] X. Zhang, L. Liu, B. Wiwatanapataphee, Y. Wu, The eigenvalue for a class of singular $p$-Laplacian fractional differential equations involving the Riemann-Stieltjes integral boundary condition, Appl. Math. Comput. 235 (2014), 412-422.

[3] G. Chai, Positive solutions for boundary value problem of fractional differential equation with $p$-Laplacian operator, Boundary Value Probl. 2012 (2012), Article ID 18.

[4] H. Lu, Z. Han, S. Sun, C. Zhang, Positive solutions for eigenvalue problems of fractional differential equations with $p$-Laplacian, Scientia Iranica B 22 (2015), 755-764.

[5] Y. Ding Y. Yang, X. Zhang, Extremal solutions for singular fractional $p$-Laplacian differential equations with nonlinear boundary conditions, Adv. Difference Equ. 2016 (2016), Article ID 201.

[6] S. Rao, Multiplicity of positive solutions for fractional differential equation with $p$-Laplacian boundary value problems, Int. J. Differ. Equ. Appl. 2016 (2016), Article ID 6906049.

[7] T. Chen, W. Liu, Z. Hu, A boundary value problem for fractional differential equation with $p$-Laplacian operator at resonance, Nonlinear Anal. 75 (2012), 3210-3217.

[8] Y. Liu, L. Lu, Multiple solutions of nonlinear fractional differential equations with $p$-Laplacian operator and nonlinear boundary conditions, Math. Slovaca 65 (2015), 79-92.

[9] L. Cheng, W. Liu Q. Ye, Boundary value problem for a coupled system of fractional differential equations with $p$ Laplacian operator at resonance, Electron. J. Differential Equations 2014 (2014), Article ID 60.

[10] T. Chen, W. Liu, An anti-periodic boundary value problem for the fractional differential equation with a $p$-Laplacian operator, Appl. Math. Lett. 25 (2012), 1671-1675.

[11] Y. Li, A. Qi, Positive solutions for multi-point boundary value problems of fractional differential equations with $p$ laplacian, Math. Methods Appl. Sci. 39 (2016), 1425-1434

[12] Z. Han, H. Lu, S. Sun, D. Yang, Positive solutions to boundary-value problems of $p$-Laplacian fractional differential equations with a parameter in the boundary, Electron. J. Differential Equations 2012 (2012), Article ID 213.

[13] T. Shen, W. Liu, X. Shen, Existence and uniqueness of solutions for several BVPs of fractional differential equations with p-Laplacian operator, Mediterranean J. Math. 13 (2016), 4623-4637.

[14] L. Hu, S. Zhang, On existence results for nonlinear fractional differential equations involving the $p$-Laplacian at resonance, Mediterranean J. Math. 13 (2016), 955-966. 
[15] Y. Liu, L. Lu, Multiple solutions of nonlinear fractional differential equations with $p$-Laplacian operator and nonlinear boundary conditions, Math. Slovaca 65 (2015), 79-92.

[16] X. Guo, Existence of unique solution to switchedfractional differential equations with $p$-Laplacian operator, Turkish J. Math. 39 (2015), 864-871.

[17] C. Yuan, Multiple positive solutions for $(n-1,1)$-type semipositone conjugate boundary value problems of nonlinear fractional differential equations, Electron. J. Qual. Theory Differ. Equ. 2010 (2010), Article ID 36.

[18] I. Podlubny, Fractional Differential Equations, Mathematics in Science and Engineering, 198, Academic Press, San Diego, (1999)

[19] S. Samko, A. Kilbas, O. Marichev, Fractional Integrals and Derivatives: Theory and Applications, Gordon and Breach, Switzerland, (1993) 